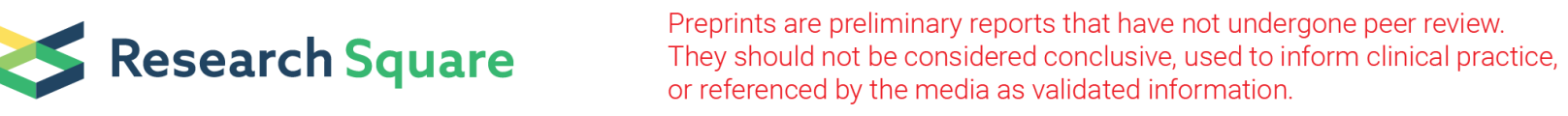

\title{
Identification of the Window Period Concurrent Infection with Syphilis and HIV: A Longitudinal Case Study
}

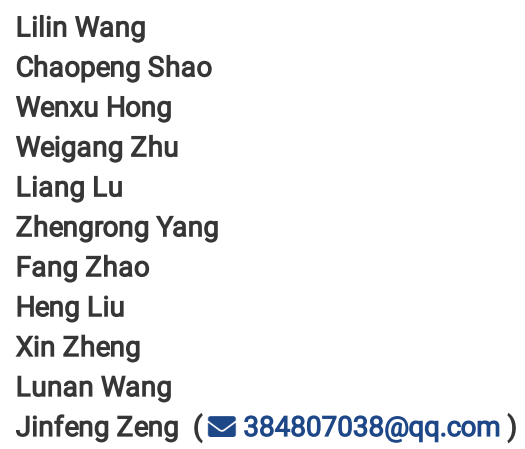




\begin{abstract}
BACKGROUND: Due to the low incidence of concurrent human immunodeficiency virus (HIV) and syphilis infection identified during the window period, little is known about the clinical manifestations, diagnosis, and treatment efficacy at very early stages.
\end{abstract}

METHODS: One longitudinal study was conducted in a 42-year-old blood donor who was concurrently infected with syphilis and HIV. This blood donor was treated with a penicillin-based regimen and early antiretroviral therapy (ART). Immunological and NAT methods were comparatively analyzed.

RESULTS: A regular male donor who had two occasions of high-risk sexual behaviors 41 and 35 days before donation(DBD) donated whole blood at the Shenzhen Blood Center. Antiretroviral therapy was initiated at the $28^{\text {th }}$ day after donation (DAD), and syphilis treatment was received at the $49^{\text {th }}$ DAD. Microbiological analysis using a $4^{\text {th }}$ generation anti-HIV ELISA and CLIA indicated a positive signal at the $6^{\text {th }}$ DAD. All NAT tests for HIV RNA were reactive except the minipool (MP) NAT of six pooled samples at $117^{\text {th }}$ DAD. The HIV viral load declined more than 4-log in copies per milliliter over 3 months, until reaching non-detectable levels at $246^{\text {th }}$ DAD. Nevertheless, HIV-1 DNA was still detectable at $403^{\text {rd }}$ DAD. Among all methods utilized, anti-TP ELISA detected syphilis infection at the earliest time. Toluidine red unheated serum test (TRUST) was negative around the $80^{\text {th }}$ DAD.

CONCLUSION: Concurrent infection with Syphilis and HIV during window period did not significantly change the sensitivity of reagents in detection nor alter the therapeutic efficacy for the treatment of both pathogens.

\title{
Introduction
}

For the last few decades, the overall incidence of syphilis has increased worldwide[1]. Syphilis often occurs with HIV infection, because these two pathogens affect the same risk groups (especially in men who have sex with men, MSM) [2]. Concurrent infection with Syphilis and HIV may change the course of virological and immunological response from a single pathogen infection. In addition, many questions, such as clinical presentation, diagnosis, and treatment for concurrent infection remain to be defined. In theory, infection by one pathogen may facilitate the infection or worsen the progression of the other pathogen. For example, syphilis may facilitate HIV transmission by producing genital ulcer to increase viral load in both semen and vaginal secretions or increase the susceptibility of the individual to HIV by decreased $C D 4^{+}$cell counts $[3,4]$. Fortunately, syphilis can be successfully treated and HIV can be clinically cured if detected at early stages [5]. However, antiretroviral therapy (ART) during acute HIV infection (AHI) may compromise HIV detction because HIV-specific antibodies, antigens, and DNA/RNA are usually altered following ART [6, 7].

The Window period (WP) infection refers to the duration after infection and before it was detected by various markers, such as HIV p24 antigen or syphilis IgM antibodies. To the best of our knowledge, concurrent WP infection by syphilis and HIV has been rarely reported due to the difficulty in tracking those infections. In this longitudinal study of a single case Syphilis/HIV co-infection, we performed a comprehensive analysis of early diagnostic results by different reagents and immune activation, inflammation, serologic ,virological responses and pathogen clearance.

\section{Case Presentation Blood Donor Information}

On April 6, 2018 (i.e. 41 and 35 days after first and second high-risk sexual behavior/exposure, respectively), a 43-year-old man donated whole blood at the Shenzhen Blood Center as his 19th visit for blood donation. Both donor questionnaire and medical interview before donation did not identify any infection risk (in particular, the donor denied any HIV risk). Moreover, regular physical examination did not reveal any abnormality. Laboratory test for donated blood revealed HIV RNA reactivity using the individual donation (ID) NAT, while the $3^{\text {rd }}$ and $4^{\text {th }}$ generation anti-HIV enzyme-linked immunosorbent (ELISA) assays were negative. Although the anti-TP reagent 1 ELISA presented the earliest positive signal for syphilis infection, other serologic screening assays were negative. During the follow-up period, the donor admitted having unprotected insertive anal intercourse twice with other men 41 and 35 days before donation(DBD). Ulceration of the external genitalia was noticed at the $28^{\text {th }}$ DBD. Accordingly, ART and penicillin-based syphilis treatment was initiated at the $28^{\text {th }}$ and $49^{\text {th }}$ DAD, respectively. We followed this donor eleven times over one year and written informed consent was obtained each time. Whole blood was collected in EDTA 2K tubes. Plasma and PBMCs were separated within $6 \mathrm{hrs}$ after collection and stored at $-80^{\circ} \mathrm{C}$ freezer until future use. Various tests were performed (see below) and the results were analyzed retrospectively.

\section{Blood screening algorithm}

Plasma samples were initially screened by two ELISA reagents and one NAT, as per the policy of batch release in China. Donor samples were individually screened for HIV-1, HCV, and HBV on the TIGRIS System using Procleix Ultrio Plus Assay (Grifols Diagnostic Solutions Inc., Emeryville, CA, USA). The following screening assays were used: HBsAg (Murex, DiaSorin S.p.A UK Branch, Dartford, UK), HBsAg (Beijing Wantai Biological Pharmacy Enterprise Co., Ltd., Beijing, China), anti-HCV (Ortho-Clinical Diagnostics, Raritan, NJ, USA), anti-HCV (Lizhu Pharmaceutical Trading Co., Ltd., Zhuhai, China), $4^{\text {th }}$ generation Genscreen ULTRA HIV Ag-Ab assay reagent 1 (Bio-Rad, Marnes-la-Coquette, France), $3^{\text {rd }}$ generation anti-HIV reagent 2 (Beijing Wantai Biological Pharmacy Enterprise Co., Ltd.), anti-TP reagent 1 (DiaSorin S.p.A UK Branch), and anti-TP reagent 2 (Lizhu Pharmaceutical Trading Co., Ltd.). Reactive samples were retested in duplicate by ELISA. Samples were considered HIV-seronegative if both retests were nonreactive; those with one of two reactive retests were considered repeat reactive.

\section{Supplementary serological tests}


The first plasma donation and follow-up samples were further tested by WB (version 2.2, MP-Biomedical, Singapore), Elecsys HIV combi ECLIA (Roche Diagnostics GmbH, Penzberg, Germany), Colloidal Gold Immunochromatographic Assay (Kehua Bio-Engineering Co., Ltd., Shanghai, China), and Alere Determine $^{T M}$ HIV1/2 (Alere Medical Co. Ltd., Matsudo-shi, Chiba-ken, Japan) following routine procedures.

\section{Supplementary NATs}

\section{MP-NAT}

The COBAS TaqScreen MPX Test, version 2.0 (MPX 2.0; Roche Molecular Systems) was used with the simulated pooling of samples in our study. The HIV WP blood donor plasma, as well as follow-up blood plasma samples without dilution and 1:6 or 1:12 dilutions with negative plasma, were tested individually using Roche TaqScreen assays.

\section{ID-NAT}

HIV WP blood donor plasma, as well as follow-up blood plasma samples, were tested with both the Ultrio Plus Assay and the Procleix Ultrio Elite assay kit (Grifols Diagnostic Solutions Inc.), as per the manufacturers' instructions.

\section{HIV RNA Quantitative assay}

Viral loads were determined using the Roche COBAS AmpliPrep/COBAS TaqMan HIV-1 Test, version 2.0 (Roche). The minimum detection limit of HIV-1 RNA is 20 copies/mL.

\section{HIV DNA Quantitative assay}

The HIV-1 Virus DNA Detection Kit (Genetic Biotek, Tianjin, China) detects HIV-1 virus DNA, which is integrated into the human genome of white cells. This kit uses pyrophosphorolysis activated polymerization technology to specifically amplify highly conserved regions within the HIV-1 virus DNA genome[8].

\section{CD4 ${ }^{+}$T cell counting}

The $\mathrm{CD} 4^{+} \mathrm{T}$ cell count from whole blood samples was determined by standard flow cytometry procedures, using a Trucount tube on a FACSCalibur instrument (BD Biosciences, San Jose, CA, USA).

\section{Results}

\section{Serological data}

According to a 4th generation anti-HIV ELISA and CLIA, blood specimen was positive at the 6th DAD. Positiveness was also detected at the 26th DAD after using a 3rd generation anti-HIV ELISA and HIV Rapid Test. After early ART treatment, the test signal value obtained with the 4th generation anti-HIV ELISA and CLIA showed a slight decline (Table 1 ). However, both 3rd generation anti-HIV ELISA and HIV Rapid Test did not show an apparent reduction of this same detection value (Table 1 ). HIV WB was positive starting at the 42nd DAD. Of all methods used, the anti-TP reagent 1 ELISA showed highest efficiency for the detection of early syphilis infection. The time for a successful serological response after syphilis treatment was reached around the 80th DAD. The results of the serological tests after early treatment of concurrent Syphilis/HIV, during the window period infection are summarized in Table 1. 
Table 1

Serological test and nucleic acid test results before and after early treatment of

\begin{tabular}{|c|c|c|c|c|c|c|c|c|c|c|}
\hline \multirow{2}{*}{$\begin{array}{l}\text { Number } \\
\text { of } \\
\text { follow- } \\
\text { up }\end{array}$} & \multirow{2}{*}{$\begin{array}{l}\text { Days after } \\
\text { donation(DAD) }\end{array}$} & \multicolumn{2}{|c|}{ ELISA anti-HIV } & \multicolumn{2}{|c|}{ ELISA anti-TP } & \multirow[t]{2}{*}{ HIV WB } & \multirow[t]{2}{*}{ TPPA(-) } & \multirow[t]{2}{*}{ Trust(-) } & \multicolumn{2}{|l|}{ HIV Rapid Test } \\
\hline & & $\begin{array}{l}\text { anti- } \\
\text { HIV R } \\
1\end{array}$ & $\begin{array}{l}\text { anti- } \\
{ }_{2}^{\text {HIV R }}\end{array}$ & $\begin{array}{l}\text { anti- } \\
\text { TP R }\end{array}$ & $\begin{array}{l}\text { anti- } \\
\text { TP R } \\
2\end{array}$ & & & & $\begin{array}{l}\text { Colloidal Gold } \\
\text { Immunochromatographic } \\
\text { Assay }\end{array}$ & $\begin{array}{l}\text { Alere } \\
\text { Dete } \\
\text { TM } \\
\text { HIV1 }\end{array}$ \\
\hline F0 & 0 & 0.31 & 0.03 & 1.76 & 0.35 & - & $\varangle 1: 80$ & - & - & - \\
\hline F1 & 6 & 6.83 & 0.01 & 1.6 & 0.73 & p24 & $₫ 1: 80$ & - & - & - \\
\hline F2 & 10 & 7.92 & 0.02 & 2.19 & 1.23 & p24 & $\otimes 1: 80$ & - & - & - \\
\hline F3 & 26 & 10.34 & 24.59 & 3.62 & 1.82 & p24,gp160 & + & - & + & + \\
\hline \multicolumn{11}{|c|}{ HIV ART at 28 DAD } \\
\hline F4 & 31 & 10.87 & 23.62 & 8.77 & 4.76 & p24,gp160 & + & + & + & + \\
\hline F5 & 42 & 8.78 & 24.59 & 11.24 & 12.45 & p24,gp120,gp160 & + & + & ++ & ++ \\
\hline \multicolumn{11}{|c|}{ Syphilis treatment at 49 DAD } \\
\hline F6 & 51 & 10.55 & 24 & 11.03 & 10.41 & p17,p24,gp120,gp160 & + & + & ++ & ++ \\
\hline F7 & 80 & 10.6 & 23.81 & 6.93 & 6.97 & p17,p24,gp120,gp160 & + & - & ++ & ++ \\
\hline F8 & 117 & 11.45 & 25 & 9.71 & 12.74 & p17,p24,gp120,gp160 & + & - & ++ & ++ \\
\hline F9 & 246 & 10.97 & 25 & 7.33 & 15.58 & p17,p24,gp120,gp160 & $1: 80$ & - & ++ & ++ \\
\hline F10 & 345 & 10.83 & 25 & 6.96 & 15.17 & p17,p24,gp41,gp120,gp160 & $1: 80$ & - & ++ & ++ \\
\hline F11 & 403 & 10.83 & 25 & 7 & 15.24 & p17,p24,gp41,gp120,gp160 & $1: 80$ & - & ++ & ++ \\
\hline
\end{tabular}

$\mathrm{F}=$ follow-up sample, $\mathrm{F} 0$ = donation sample.

$R 1$ = reagent $1 ; R 2$ = reagent 2.

ND, not determined (Insufficient sample volume, unable to contact, or other reasons)

NDD, When the test result is "no HIV-1DNA detected", it is interpreted as: HIV-1DNA is lower than 10 copies/mL and cannot be detected, or there is no HIV-1 DI NRD, When the test result is "no HIV-1RNA detected", it is interpreted as: HIV-1RNA is lower than 20 copies/mL and cannot be detected, or there is no HIV-1 RN When the test result is $<2.00 \mathrm{E}+01$, it is interpreted as: HIV-1RNA was detected, but below the detection limit $(20 \mathrm{copies} / \mathrm{mL})$.

One copy of HIV-1 RNA is equivalent to $1.7+0.1$ international unit (IU).

\section{HIV-related nucleic acid test}

TMA and PCR methods have been routinely chosen for blood screening at our Center. At the first blood donation made by the donor, all NAT methods for HIVRNA detection were reactive. However, MP NAT of six samples (6 MP) were nonreactive at the 117th DAD. After early ART, HIV viral loads declined more than 4log in copies per milliliter over 3 months, until reaching undetectable levels of HIV RNA NAT or HIV RNA viral load at the 246th DAD. Nevertheless, HIV-1 DNA was still detectable at the 403rd DAD even after an apparent decline. Results of the nucleic acid tests before and after early treatment of this donor are listed in Table 1.

\section{CD4 lymphocyte count and CD4/CD8 ratio}

As shown in Table 2, both CD4 count and CD4/CD8 ratio decreased significantly during acute infection. Treatment with ART and penicillin rescued the fall of both the CD4 count and CD4/CD8 ratio, despite a fluctuation at the 345 th DAD was observed (Table 2). 
Table 2

Trend of CD4/CD8 ratio after early treatment of HIV/TP coinfected blood donor

\begin{tabular}{|lllll|}
\hline Number of follow-up & Days after donation(DAD) & CD8 T cell & CD4 T cell & ratio \\
\hline F0 & 0 & ND & ND & ND \\
\hline F1 & 6 & ND & ND & ND \\
\hline F2 & 10 & 123 & 236 & 1.91 \\
\hline F3 & 26 & 716 & 358 & 0.50 \\
\hline HIV ART at 28 DAD & & & & \\
\hline F4 & 31 & 693 & 384 & 0.55 \\
\hline F5 & 42 & ND & ND & ND \\
\hline Syphilis treatment at 49 DAD & & & \\
\hline F6 & 51 & 418 & 445 & 0.94 \\
\hline F7 & 80 & 377 & 450 & 1.19 \\
\hline F8 & 117 & 396 & 594 & 1.50 \\
\hline F9 & 246 & 254 & 525 & 2.07 \\
\hline F10 & 345 & 199 & 370 & 1.86 \\
\hline F11 & 403 & 296 & 522 & 1.76 \\
\hline ND, not determined (Insufficient sample volume, unable to contact, or other reasons) \\
\hline
\end{tabular}

\section{Discussion}

Here we present a comprehensive longitudinal study, including the observation of clinical manifestation, diagnosis and treatment, based on a concurrent Syphilis/HIV infection identified during window period. The main purpose of this longitudinal study is to evaluate whether the diagnostic power (efficiency) of different test methods (both serological and nucleic-acid tests) is compromised in Syphilis/HIV co-infected donors before and after clincal treatment.

Disease progression may be altered in co-infected or post-treatment individuals. Previous research has shown that, in addition to functional defects of macrophages, HIV-induced breakdown of cell-mediated and humoral immunity alters the course of syphilis progression. The progression of syphilis is usually faster in HIV patients than in those not infected by this virus [9]. In addition, ART usually modulates host immunity, by a series of mechanisms that are not fully understood. Although the median length of time from the initial infection to a positive syphilis serological test is 10 days (4-60 days), this median time can increase up to 32 days in individuals where an initial ELISA test is negative [10].

Different tests vary in sensitivity. In this case report, the length of time between the ulceration of the external genitalia (identified at the 28th DBD) to a positive serological test was 0,6 and 10 DAD using anti-TP reagent 1, anti-TP ECLIA and anti-TP reagent 2 tests, respectively. These data values were within the ranges observed in mono-infected syphilis patients [10]. These results indicated that HIV/syphilis co-infection does not compromise the diagnostic power (efficiency) of different test methods for the detection of syphilis. However, syphilis treatment usually has a lower response rate in HIV-infected individuals as shown by slower decrease in non-treponemal titers [11]. In our case, we observed that both IgG and IgM levels were decreased following syphilis treatment and a successful serological response was obtained at the 80th DAD. This data demonstrate that HIV/Syphilis co-infeceted individuals respond well to syphilis treatment although we are not quite sure whether HIV-infection weakens the response rate or not.

A positive response to syphilis treatment requires monitoring of nontreponemal antibody titers, with more than 4-fold decline and/or seroreversion to nonreactive titers. As reported, $15-25 \%$ of patients treated during the primary stage revert to serologically non-reactive after 2-3 years [12]. The majority of HIVnegative patients with early syphilis failed to have seroreversion at 12 months [13]. Seroreversion at six months after treatment appears to be similar among all primary syphilis patients [14]. In our study, a successful serological response to syphilis treatment was reached 80th DAD. Our data is in agreement with previous findings that timely treatment of syphilis is highly effective, even in co-infection patients [15]. Some previous studies have also evaluated the association of HIV status with serologic outcomes, after syphilis treatment, by comparing patients with and without HIV infection [16, 17]. Unfortunately, the results of these particular studies are inconsistent, so it remains unclear whether HIV-infected individuals are less likely to achieve serologic clearance of syphilis. At least in part, these contrasting results might be related to (i) the variability in the stages of syphilis and HIV infection status, and (ii) an inconsistent definition of serological non-response.

Identificaiton of the WP infections is difficult. As recommended by the UK national guidelines, the 4th generation of HIV laboratory tests for HIV p24 detection is more applicable for shorter WP as compared to rapid tests and 3rd generation ELISA, which typically reduces WP to 1.56 and 5.32 days, respectively [18, 19]. According to BASHH/EAGA Position Statement on HIV Window Period (November 2014), a negative result of the 4th generation testing obtained four weeks after exposure is highly likely to exclude HIV infection. However, based on our current study, the window period appears to be longer in HIV/syphilis coinfected individual. 
In this case study, the 4th generation anti-HIV ELISA and CLIA platform provided a positive signal at the 6th DAD. It had been nearly six weeks since the last high-risk exposure of the 41st and 35th DBD. We speculate that one possible reason for this longer WP might be that syphilis infection affects the progression of humoral response to HIV. Moreover, the behavior of an insertive anal intercourse could lead to a lower concentration of receptive virus, affecting the HIV dynamics and immune response.

In 2015, the ART guidelines from the World Health Organization (WHO) recommended treatment only after HIV infection was detected [20, 21]. In addition, the early initiation of ART following infection may limit the viral burden in the presence of HIV [22]. Qualitative HIV DNA tests are preferred for confirmation at a later stage. As shown in Table 1, we observed that, after early ART treatment, the test signal from the 4th generation anti-HIV ELISA and CLIA showed slightly declining although no apparent reduction was detected by both 3rd G anti-HIV ELISA and HIV Rapid Test. This data demonstrates that, although the 4th generation ELISA was the most sensitive test to detect AHI prior to ART, the 3rd generation approach was the most sensitive during treatment [7]. Furthermore, this co-infected donor responds well to ART as shown by the declined HIV viral loads until reaching undetectable levels at the 246th DAD although HIV-1 DNA was detectable by the 403rd DAD (Table 1). Therefore, in accordance with previous work, our data illustrates the critical role of early stage treatment for HIV[23]. This data also indicates that HIV DNA can still be detected, as virus reservoir, after co-infection.

According to the background information, this blood donor presented one genital ulcer (chancre) at the 28th DBD. Previous research has shown that chancres typically appear 10-90 days after exposure [24]. The window period whereby the humoral response to syphilis develops is between 1 to 4 weeks after the chancre forms in primary syphilis [25]. Apparently, co-infection appears to have limited effect towards this length of time.

Although this is a comprehensive longitudial follow-up study, our report also faces some limitations. First, the patient had two high-risk behaviors but, due to the lack of follow-up data, the specific infection time could not be determined. Second, this study is mainly focused on testing and treatment, the molecular mechanism(s) related to the effect of coinfection towards the immune system remains in-depth investigation. Nevertheless, based on this rare case study, we strongly believe that our current data will serve as an useful background information for future research.

\section{Conclusions}

Based on our current study, the window period of HIV appears to be longer but syphilis window period time appears to have limited effect in HIV/syphilis coinfected individual. HIV/Syphilis co-infeceted individuals respond well to syphilis treatment although we are not quite sure whether HIV-infection weakens the response rate or not. In conclusion, concurrent infection with Syphilis and HIV during window period did not significantly change the sensitivity of reagents in detection nor alter the therapeutic efficacy for the treatment of both pathogens.

\section{Abbreviations}

AIDS = acquired immunodeficiency syndrome; $A R T$ = antiretroviral therapy; DAD = day after donation; DBD = day before donation; ELISA = enzyme linked immunosorbent assay; HIV = human immunodeficiency virus; ID = individual donation; MP = mini-pool; NAT = nucleic acid testing; TRUST = tolulized red unheated serum test; TPPA = treponema pallidum passive particle agglutination test; $S N R=$ signal to noise ratio; $W B=$ western blot; WP = window period.

\section{Declarations}

\section{Acknowledgments}

The authors wish to thank Shenzhen Center for Disease Control and Prevention for providing HIV WB for this study and Shenzhen Third People's Hospital for providing early treatment for the blood donor. The authors gratefully acknowledge the blood donor who volunteers to participate in this Study. We are indebted to Professor Limin Chen from the Institute of Blood Transfusion, Chinese Academy of Medical Sciences and Peking Union Medical College for his critical review of this manuscript.

\section{Funding}

This study was supported by grants from the Medical Scientific Research Foundation of Guangdong Province (grant no. A2020259), the Shenzhen Science and Technology Plan Project (grant no. JCYJ20180302153439758), Shenzhen Medical Research Funding (grant no. SZGW2018008) and the Sanming Project of Medicine in Shenzhen (grant no. SZSM201811092).

\section{Availability of data and materials}

The datasets used and/or analyzed during this study are available from the corresponding author on reasonable request.

\section{Authors' contributions}

Lilin Wang wrote the paper with the participation of Chaopeng Shao and Wenxu Hong $\rrbracket$

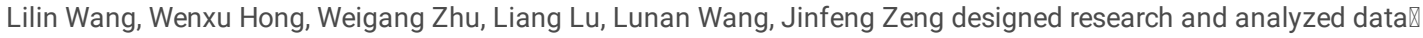

Zhengrong Yang contributed HIV WB test囚 
Fang Zhao provided early treatment for the blood donor】

Lilin Wang, Xiaoxuan Xu, Wen Xiong performed research.

\section{Competing interests}

The authors declare that they have no competing interests.

\section{Ethics approval and consent to participate}

This study was approved by the Ethics Committee of Shenzhen Blood Center.

\section{Patient consent for publication}

Not applicable.

\section{Competing interests}

The authors declare that they have no competing interests.

\section{References}

1. Hook EW. Syphilis. Lancet. 2017;389(10078):1550-7.

2. Traeger MW, Cornelisse VJ, Asselin J, et al. Association of HIV Preexposure Prophylaxis With Incidence of Sexually Transmitted Infections Among Individuals at High Risk of HIV Infection. JAMA. 2019;321(14):1380-90.

3. Buchacz K, Patel P, Taylor M, et al. Syphilis increases HIV viral load and decreases CD4 cell counts in HIV-infected patients with new syphilis infections. AIDS. 2004;18(15):2075-9.

4. Kofoed K, Gerstoft J, Mathiesen LR, Benfield T. Syphilis and human immunodeficiency virus (HIV)-1 coinfection: influence on CD4 T-cell count, HIV-1 viral load, and treatment response. Sex Transm Dis. 2006;33(3):143-8.

5. French P, Gomberg M, Janier M, Schmidt B, van Voorst Vader P, Young H. IUSTI: 2008 European Guidelines on the Management of Syphilis. Int J STD AIDS. 2009;20(5):300-9.

6. Cohen MS, Chen YQ, McCauley M, et al. Antiretroviral Therapy for the Prevention of HIV-1 Transmission. N Engl J Med. 2016;375(9):830-9.

7. de Souza MS, Pinyakorn S, Akapirat S, et al. Initiation of Antiretroviral Therapy During Acute HIV-1 Infection Leads to a High Rate of Nonreactive HIV Serology. Clin Infect Dis. 2016;63(4):555-61.

8. Liu Q, Sommer SS. PAP: detection of ultra rare mutations depends on $\mathrm{P}^{*}$ oligonucleotides: "sleeping beauties" awakened by the kiss of pyrophosphorolysis. Hum Mutat. 2004;23(5):426-36.

9. Johns DR, Tierney M, Felsenstein D. Alteration in the natural history of neurosyphilis by concurrent infection with the human immunodeficiency virus. $\mathrm{N}$ Engl J Med. 1987;316(25):1569-72.

10. Wheeler HL, Agarwal S, Goh BT. Dark ground microscopy and treponemal serological tests in the diagnosis of early syphilis. Sex Transm Infect. 2004;80(5):411-4.

11. Ghanem KG, Erbelding EJ, Wiener ZS, Rompalo AM. Serological response to syphilis treatment in HIV-positive and HIV-negative patients attending sexually transmitted diseases clinics. Sex Transm Infect. 2007;83(2):97-101.

12. Romanowski B, Sutherland R, Fick GH, Mooney D, Love EJ. Serologic response to treatment of infectious syphilis. Ann Intern Med. 1991;114(12):1005-9.

13. Seña AC, Wolff M, Behets F, et al. Rate of Decline in Nontreponemal Antibody Titers and Seroreversion After Treatment of Early Syphilis. Sex Transm Dis. 2017;44(1):6-10.

14. Kim JI, Park JH, Choi JY, Lee GY, Kim WS. Serologic Response to Treatment in Human Immunodeficiency Virus-Negative Syphilis Patients Using Automated Serological Tests: Proposals for New Guidelines. Ann Dermatol. 2017;29(6):768-75.

15. Blencowe H, Cousens S, Kamb M, Berman S, Lawn JE. Lives Saved Tool supplement detection and treatment of syphilis in pregnancy to reduce syphilis related stillbirths and neonatal mortality. BMC Public Health. 2011;11 Suppl 3:S9.

16. Jinno S, Anker B, Kaur P, Bristow CC, Klausner JD. Predictors of serological failure after treatment in HIV-infected patients with early syphilis in the emerging era of universal antiretroviral therapy use. BMC Infect Dis. 2013;13:605.

17. Kenyon C, Osbak KK, Apers L. Repeat Syphilis Is More Likely to Be Asymptomatic in HIV-Infected Individuals: A Retrospective Cohort Analysis With Important Implications for Screening. Open Forum Infect Dis. 2018;5(6):ofy096.

18. Weber B, Fall EH, Berger A, Doerr HW. Reduction of diagnostic window by new fourth-generation human immunodeficiency virus screening assays. J Clin Microbiol. 1998;36(8):2235-9.

19. Weber B, Orazi B, Raineri A, et al. Multicenter evaluation of a new 4th generation HIV screening assay Elecsys HIV combi. Clin Lab. 2006;52(9-10):463-73. 
20. Lundgren JD, Babiker AG, Gordin F, et al. Initiation of Antiretroviral Therapy in Early Asymptomatic HIV Infection. N Engl J Med. 2015;373(9):795-807.

21. Lifson AR, Grund B, Gardner EM, et al. Improved quality of life with immediate versus deferred initiation of antiretroviral therapy in early asymptomatic HIV infection. AIDS. 2017;31(7):953-63.

22. Siliciano JD, Kajdas J, Finzi D, et al. Long-term follow-up studies confirm the stability of the latent reservoir for HIV-1 in resting CD4+ T cells. Nat Med. 2003;9(6):727-8.

23. Saag MS, Benson CA, Gandhi RT, et al. Antiretroviral Drugs for Treatment and Prevention of HIV Infection in Adults: 2018 Recommendations of the International Antiviral Society-USA Panel. JAMA. 2018;320(4):379-96.

24. Singh AE, Romanowski B. Syphilis: review with emphasis on clinical, epidemiologic, and some biologic features. Clin Microbiol Rev. 1999;12(2):187-209.

25. French P. Syphilis. BMJ. 2007;334(7585):143-7.

\section{Supplementary Files}

This is a list of supplementary files associated with this preprint. Click to download.

- Coinfectioncoveringletter2020.6.22ART.docx 